\title{
Scale-free aggregation and interface fluctuations of cancer clusters in cancer-endothelial cell mixtures: From the dilute state to confluent monolayer
}

\author{
Chun-Yu Liu ๑, Hsiang-Ying Chen, and Lin I๑ \\ Department of Physics and Center for Complex Systems, National Central University, Jhongli, Taiwan 32001, Republic of China
}

(Received 23 July 2020; accepted 4 August 2021; published 23 August 2021)

\begin{abstract}
We experimentally investigate the multiscale dynamical and scaling behaviors of the morphology, motion, and area distribution of segregated cancer clusters of proliferating cancer-endothelial cell mixtures, from the initial randomly distributed dilute state to the jammed confluent monolayer state. Cancer cells (CCs) have higher motilities than endothelial cells (ECs) and weaker CC-EC and CC-CC cohesive couplings than EC-EC coupling. It is found that, with increasing waiting time $t_{w}$, CCs proliferate, move, and aggregate into clusters with fractal cluster boundaries, increasing averaged cluster area, and the gradual transition to the self-similar power-law distribution of single cluster areas. In CC clusters, multiscale turbulentlike motion and the interaction with surrounding ECs are the keys for self-similar multiscale spatial fluctuations of the velocities and fractal structure of CC cluster boundaries. The larger CC cluster allows stronger longer-length fluctuations. It causes the increasing fractal dimension and scaling exponents of the relative motion of $\mathrm{CC}$ cluster boundaries, which can be suppressed by proliferation-induced crowding and dynamical slowing down.
\end{abstract}

DOI: 10.1103/PhysRevResearch.3.L032050

Fluctuating interfaces ubiquitously occur in various nonequilibrium media [1]. The interplay of different coherences generated by different mutual couplings on the two sides of the interface, and the disorder generated by passive/active forces, stochastic agitations, or mass inputs, could generate self-similar multiscale spatiotemporal interface fluctuations [1]. Solid-liquid fronts in melting or solidification [1-3], paper burning or wetting fronts [1,4,5], fronts of diffusion-limited aggregations [1,6], and invading interfaces of mobile bacteria into demobilized bacteria [7] are a few good examples.

For passive or active binary mixtures, the same type of species could segregate into clusters [8-17]. For example, for binary mixtures at the continuous limit, clusters with smooth boundaries governed by surface tension can be formed through spinodal decomposition $[8,12]$. However, the generic dynamical behaviors of aggregation and fluctuating cluster boundaries of binary mixtures down to the microscopic level, especially for active cell systems, remain elusive.

The cell system is a coupled active system exhibiting motions over a wide range of scales, which are important in many biological processes such as embryogenesis [18], tumorigenesis [19], and cancer metastasis [20-22]. Comparing with coupled passive systems, the additional self-propelled motion, unfrozen proliferation, deformable cell boundary, etc., enrich the structural and dynamical behaviors [23-33]. For example, a proliferation-induced liquid-glassy transition, and collective

Published by the American Physical Society under the terms of the Creative Commons Attribution 4.0 International license. Further distribution of this work must maintain attribution to the author(s) and the published article's title, journal citation, and DOI. motion in the form of multiscale swirls, turbulence, follow the leader streaming, and chain-type collective migration, have been demonstrated in monotype cell systems [23-28]. The fractal-like distribution of cell nuclei in the arrested epithelial cell clusters with highly suppressed motility by tuning growth factors was also reported [33].

For cell mixtures, the behaviors of structure and motion can be further enriched [10-17,34]. The segregation of the same type of cells into clusters due to the different motilities and different cell-cell couplings of the two different types of cells is a special feature. Numerous works reported that tumors exhibit scale-free fractal patterns and fractal boundaries. However, they were measured from static histological images of stained tissues without addressing dynamical behaviors [35-37]. Past studies on the dynamical behaviors of other binary cell mixtures have mainly focused on finding the temporal evolutions of the sizes of dominant segregated clusters [10-14] and the degrees of segregation [15-17], by tuning cell motility and coupling. The recent experimental work on the invasion of a small fraction of more mobile cancer cells (CCs) into the confluent monolayer endothelial cells (ECs) demonstrated that the aggregation of CCs into a larger cluster enhances turbulentlike $\mathrm{CC}$ motion and rejuvenates the jammed motion of the surrounding ECs induced by proliferation [23].

Nevertheless, the multiscale structural and dynamical behaviors of aggregation and fluctuating cluster boundaries, and their correlation with turbulentlike cell motions of the proliferating monolayer binary cell mixture, from the dilute state with similar number fractions of the two types of cells with different motilities, remain unexplored. Here, using the CC-EC mixture as an example, the above intriguing issues are experimentally unraveled. We demonstrate the gradual aggregation of individual CCs and small CC clusters into larger 
(a)
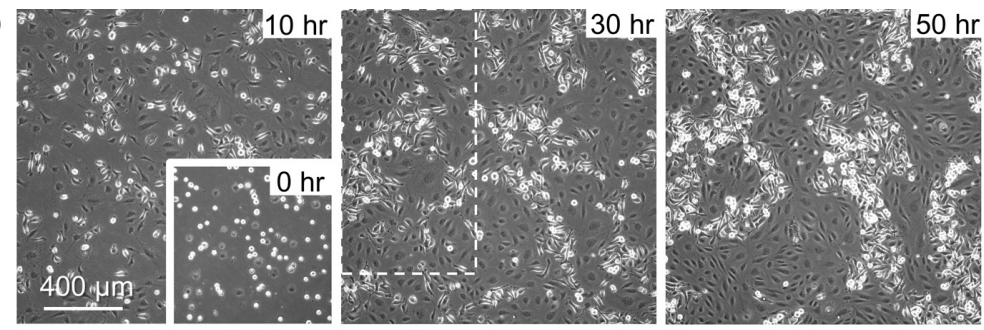

(c) 10

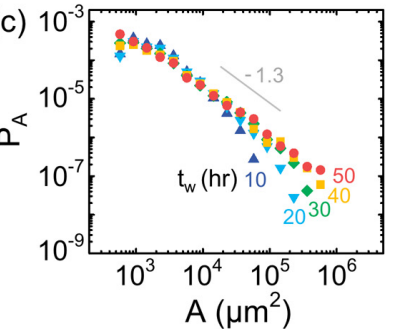

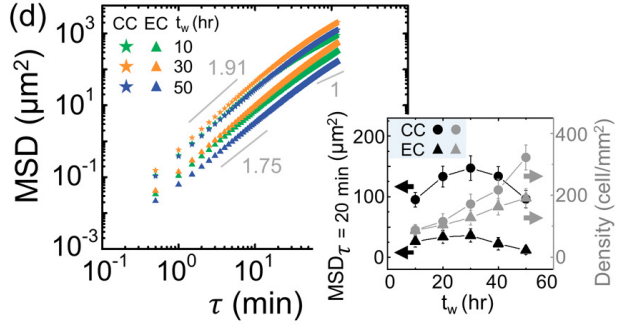

(b)

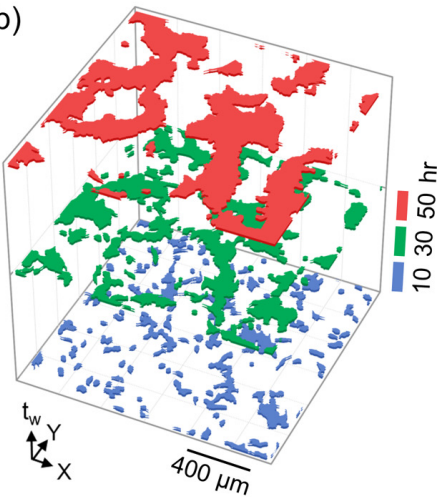

FIG. 1. (a) Phase contrast images of the CC-EC mixture at $t_{w}=0,10,30$, and $50 \mathrm{~h}$, respectively. CCs have brighter cell boundaries. (b) Spatiotemporal distribution of CC clusters from the same region as that of (a). (c) Probability distribution functions $P_{A}$ of the CC cluster area $A$ at different $t_{w}$, showing the stretching of the descending tails to the scale-free power-law decay with the scaling exponent equaling -1.3 , as $t_{w}$ increases. (d) MSDs of CCs and ECs at different $t_{w}$ from the Lagrangian trajectory of each element, with the numbers indicating the scaling exponents of the reference straight gray line. Inset: Temporal evolutions of cell density (cell number divided by image area) and MSD over the 20-min interval of CCs and ECs. Straight lines between data points in the inset are used as guides for the eye.

$\mathrm{CC}$ clusters, associated with the transition to the scale-free $\mathrm{CC}$ cluster size distribution, and illustrate how the turbulent $\mathrm{CC}$ motions lead to the observed scale-free fluctuations of the velocity and positions of CC boundaries, associated with the dynamical slowing down through proliferation-induced crowding.

We use bone marrow metastasis nasopharyngeal carcinoma cells (CCs) and human umbilical vein endothelial cells (ECs) [38]. The CC has a higher motility than that of the EC. The strengths of mutual cell-cell adhesion from strong to weak follow the order: EC-EC (strong VE-cadherin junctions), CC-CC (weak E-cadherin junctions), and EC-CC (no junctions) [38]. The initial CC/EC number ratio is 1.5 (the initial packing densities of CCs and ECs are 88 and $58 \mathrm{cell} / \mathrm{mm}^{2}$, respectively), for easily forming large $\mathrm{CC}$ clusters separated by surrounding ECs [38]. The waiting time $t_{w}$ is set to 0 when randomly distributed CCs and ECs simultaneously land on a collagen-coated substrate [Fig. 1(a)]. The spatiotemporal dynamics of the CC-EC monolayer is recorded by a time-lapse phase contrast microscope. The velocity fields are obtained using particle image velocimetry (PIV) [39]. More experimental details of cell incubation, PIV, CC cluster boundary identification and Lagrangian trajectory tracking can be found in the Supplemental Material (SM) [38].

Figure 1(a) shows the phase contrast images of the CC-EC mixture at $t_{w}=0,10,30$, and $50 \mathrm{~h}$. CCs have bright cell boundaries. Figure 1(b) shows the temporal evolution of $\mathrm{CC}$ clusters in the xyt space (also see Video 1 [38]). At $t_{w}=0 \mathrm{~h}$, the dilute CCs and ECs are mainly circular. After attaching to the substrate, cells spread and CCs gradually aggregate into clusters with irregular boundaries. After $30 \mathrm{~h}$, a confluent monolayer is formed. As demonstrated in Figs. 1(a) and 1(b), continuing cell proliferation [see the increasing cell density with $t_{w}$ in the inset of Fig. 1(d)] and aggregation increase the averaged CC cluster size. The double-logarithmic plots of $P_{A}$, the probability distribution function of the $\mathrm{CC}$ cluster area $A$, in Fig. 1(c) show the gradual stretching of the descending tails to the scale-free power-law decay with increasing $t_{w}$

Cells move in the above process. Figure 1(d) shows the curves of CC and EC mean-square displacements (MSDs) over a time interval $\tau$ from the Lagrangian trajectory of each element in the two types of cells (see SM [38] for the trajectory construction), starting in 1-h intervals after $t_{w}=10,30$, and $50 \mathrm{~h}$. All the curves with scaling exponents greater than 1 manifest superdiffusion. At each $t_{w}$, the larger MSD with the larger scaling exponent for CCs indicates the faster and more persistent motion than that of ECs. Moreover, MSD curves are upward shifted before $t_{w}=30 \mathrm{~h}$ and downward shifted after $t_{w}=30 \mathrm{~h}$. The former can be attributed to the enhanced cooperative motion through the increasing mutual coupling caused by the proliferation and aggregation into larger clusters. The latter indicates the dynamical slowing down due to proliferation-induced crowding. They can also be clearly manifested by the inset of Fig. 1(d), showing the temporal dependence of MSDs of CCs and ECs over a 20-min interval, respectively. The speeding-up/slowing-down transitions for CCs and ECs both occur around $t_{w}=30 \mathrm{~h}$, the time for cells forming a confluent cell monolayer.

CCs aggregate into clusters with irregular boundaries (Videos 1 and 2 [38]). Figure 2(a) shows the evolution of $\mathrm{CC}$ cluster boundaries, enlarged from the boxed region of Fig. 1(a) at $t_{w}=10,30$, and $50 \mathrm{~h}$. The gray areas are CC clusters at $t_{w}$ and the blue solid lines are CC cluster boundaries $1 \mathrm{~h}$ after $t_{w}$. The irregular CC cluster boundaries still fluctuate spatiotemporally even after forming the confluent cell monolayer for $t_{w} \geqslant 30 \mathrm{~h}$.

$\mathrm{CC}$ cluster boundaries exhibit a fractal structure. It can be evidenced by the power-law increase of $\langle N(r)\rangle$ with $r$ in Fig. 2(b), where $N(r)$ is the number of pairs of points 
(a)

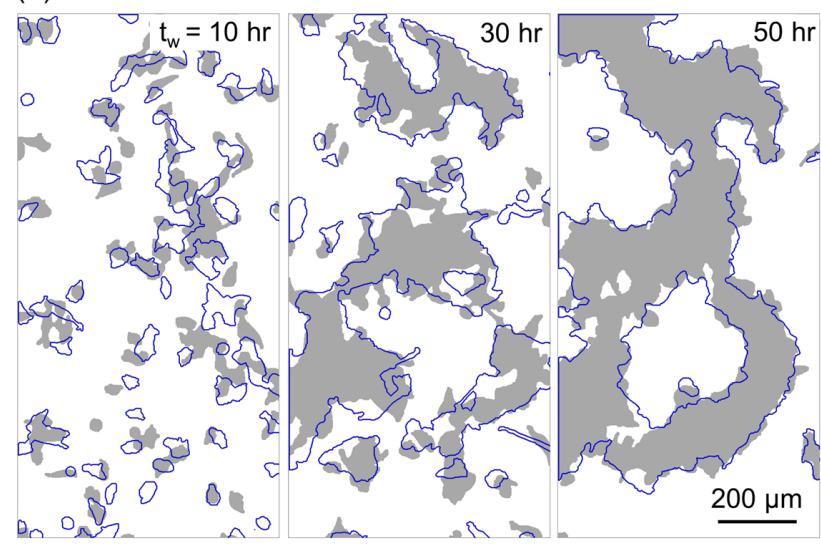

(b)

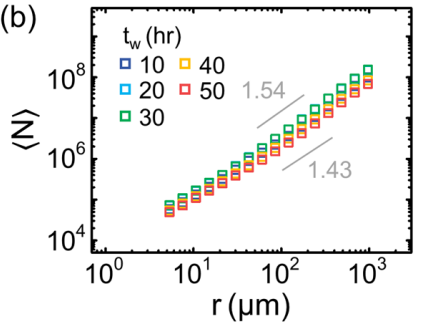

(d)

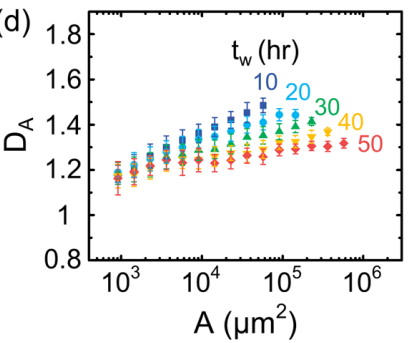

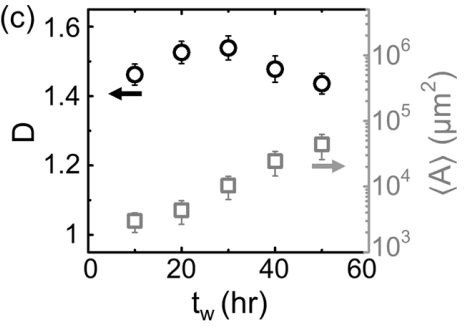

(e)

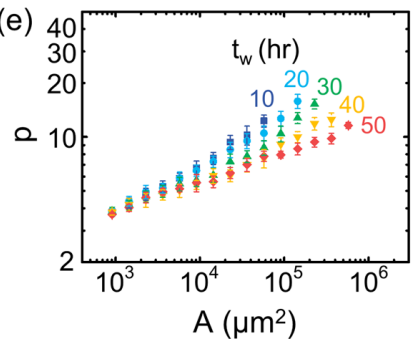

FIG. 2. (a) Evolutions of CC clusters at $t_{w}=10,30$, and $50 \mathrm{~h}$, enlarged from the boxed region of Fig. 1(a). The gray areas are CC clusters at each $t_{w}$ and the blue solid lines are CC cluster boundaries $1 \mathrm{~h}$ after $t_{w}$. (b) Averaged number of pairs of points $\langle N(r)\rangle$ with separation $r$ on the boundaries of all clusters at different $t_{w}$. The numbers by the gray lines correspond to the scaling exponents (fractal dimensions). (c) Temporal evolutions of the fractal dimension $D$ of the boundaries of all clusters and averaged cluster area $\langle A\rangle$. (d) Averaged fractal dimension $D_{A}$ of the boundaries of the single CC cluster with area $A$. (e) Shape index $p$ of the single CC cluster boundary vs CC cluster area $A$.

with a separation smaller than $r$ on the boundaries of all $\mathrm{CC}$ clusters in each image, and the average is taken over 1-h images after a fixed $t_{w}$. Namely, the structural fluctuation is statistically similar to itself on magnification over a finite range of length scales. The slope of each double-logarithmic curve corresponds to the fractal (correlation) dimension $D$, which also quantifies the effective dimensions and degrees of complexity of the irregular CC boundary [1].

Each single CC cluster boundary is also fractal (Fig. S4 [38]). Figure 2(d) shows $D_{A}$, the fractal (correlation) dimension of the boundary of the single $\mathrm{CC}$ cluster with area $A$, averaged over all those clusters from the 5-h images after each $t_{w}$. The curves of $D_{A}$ all increase with $A$. The increasing interface complexity can be contributed by the stronger fluctuations of the longer scales in the larger CC cluster, and further supported by Fig. 2(e) showing the increasing shape index $p$ with cluster area $A$. Here, $p$ is defined as the averaged ratio of the $\mathrm{CC}$ cluster boundary length to the square root of CC cluster area [25], which also measures interface irregularity. $D, D_{A}$, and $p$ show faster descents after $t_{w} \geqslant 30 \mathrm{~h}$.

We further characterize the spatial scaling behavior of velocity fluctuations along the boundary of the single cluster with area $A$ by measuring the mean-square relative velocity, MSRV $=\left\langle|\delta \mathbf{V}(r)|^{2}\right\rangle$, where $\delta \mathbf{V}(r)=\mathbf{V}_{i}-\mathbf{V}_{j}$ is the velocity difference of two elements $i$ and $j$ with separation $r$ on a $\mathrm{CC}$ cluster boundary. The average is taken over all the single CC clusters with the same area $A$ of the images within a 5-h time interval. As shown in Fig. 3(c), all the MSRV curves exhibit a power-law increase with $r$ until leveling off around $r=50 \mu \mathrm{m}$ (i.e., the velocity correlation length shown in Fig. S6 [38]). The scaling exponent $\alpha$ and the magnitude of MSRV both slightly increase with increasing $A$ from $2 \times 10^{3}$ to $3 \times 10^{4} \mu \mathrm{m}^{2}$. With increasing $t_{w}$, they first slightly rise and then decrease after $t_{w}=30$ h. $\alpha>1$ implies the superlinear nonrandom scale-free increase of MSRV with increasing $r$, contributed by the multiscale turbulent $\mathrm{CC}$ motions.

What is the origin of CC clusters with scale-free cluster size distributions and scale-free boundary fluctuations in structure and motion? In continuous passive binary mixtures, segregated patterns exhibit smooth boundaries for minimizing interfacial energy [8,9]. However, in our active binary mixture, the difference in cell motilities and intercellular coupling of CCs and ECs, and the floppy cell boundaries allow richer behaviors in structure and motion. The previous study on the invasion of randomly distributed low concentration CCs into a confluent and dense EC monolayer demonstrated the aggregation-enhanced turbulentlike multiscale $\mathrm{CC}$ motion with increasing CC cluster size [23]. This turbulent CC motion is certainly a source for the fluctuating $\mathrm{CC}$ cluster boundary.

In this study, starting from the low-density $\mathrm{CCs}$ and ECs with $1.5 / 1$ concentration ratio, CCs can aggregate into larger clusters than those in the previous study [23]. Figure 3(a) and the left panel of Fig. 3(b) enlarged from the boxed region of Fig. 3(a) show typical plots of the velocity fields color coded by velocity orientation, where the gray areas represent the areas of CC clusters. The enlarged plot in the right panel of Fig. 3(b) illustrates the trajectories over $1 \mathrm{~h}$, color coded by trajectory length. Video 2 [38], and the power-law decay of the power spectra of temporal evolutions of $\mathrm{CC}$ element Lagrangian velocities in Fig. S5 [38] further show scale-free turbulentlike CC motion. The spatial correlation length of Eulerian CC velocity increases from 55 to $60 \mu \mathrm{m}$ and then decreases to $50 \mu \mathrm{m}$ with increasing cell coupling as $t_{w}$ increases (Fig. S6 [38]). It corresponds to the scales of colored patches in the large clusters.

The motion coherence of a single CC cluster, defined as the magnitude of the mean velocity normalized by the mean speed of all the elements in the CC cluster, decreases with 
(a)

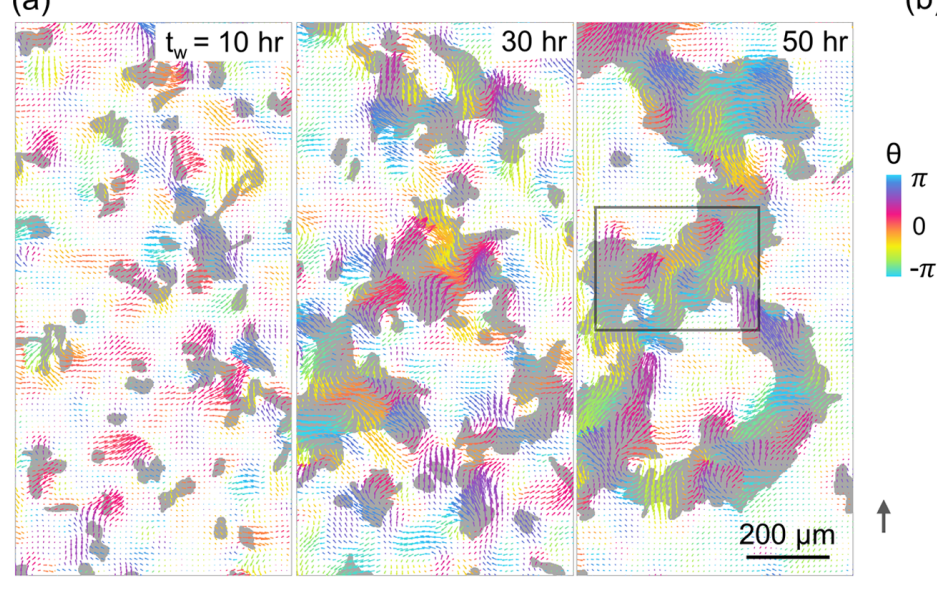

(b)
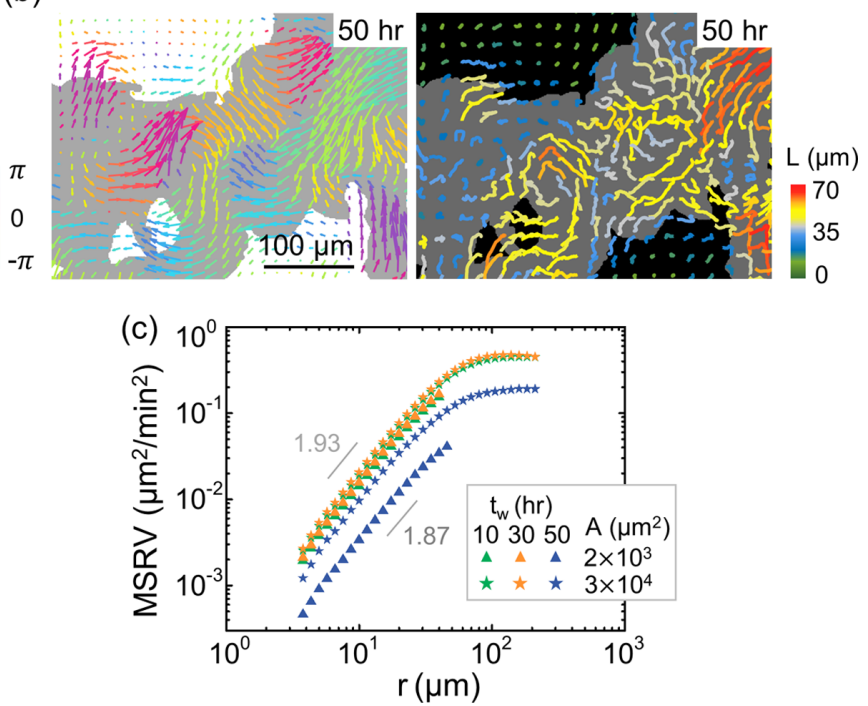

FIG. 3. (a) Velocity fields of the same region as that of Fig. 2(a), color coded by velocity directions at different $t_{w}$. The gray regions are $\mathrm{CC}$ clusters. The length of the vertical black vector at the lower right corner represents a velocity vector with speed $=2 \mu \mathrm{m} / \mathrm{min}$. (b) Left: Enlarged velocity field from the boxed region of (a) at $t_{w}=50 \mathrm{~h}$. Right: 1-h element trajectories color coded by the trajectory length $L$ of the same region as the left panel. (c) MSRV vs $r$, for clusters at $A=2 \times 10^{3}$ and $3 \times 10^{4} \mu \mathrm{m}^{2}$ and $t_{w}=10,30$, and $50 \mathrm{~h}$. The numbers by the gray lines correspond to the scaling exponents.

increasing cluster area. For the small CC cluster with a size smaller than $50 \mu \mathrm{m}$, the high motion coherence [also see the velocity vectors with similar colors (directions) in the first panel of Fig. 3(a)] can make all CCs move together and merge with other CCs to form larger clusters. In the larger $\mathrm{CC}$ cluster, more patches with different velocity orientations lead to decreasing motion coherence with increasing $A$ [see Fig. S6(c) [38]].

Note that, for the monotype CC system from the dilute state with increasing cell density through proliferation, the self-propelled cell motions suppress CCs from aggregating into clusters with dense cell packing [see Fig. S3(d) and Video 3 [38]]. However, for our binary CC-EC mixture, the weaker CC-EC coupling than CC-CC and EC-EC couplings leads to the easy formation of $\mathrm{CC}$ clusters surrounded by more strongly coupled ECs which have a lower motility than that of CCs (see Video 1 [38]). The CC cluster size can increase through the gradual merging of single $\mathrm{CCs}$ or $\mathrm{CC}$ clusters with different areas. The turbulent CC motion can also cause the splitting of a CC cluster into smaller clusters. The final power-law cluster size distribution in the later stage [Fig. 1(c)] manifests that similar dynamical rules for aggregation are followed over a certain range of scales.

For a nonequilibrium nonlinear extended system, the interplay between the order/coherence generated by mutual interaction, and the disorder excited by the stochastic drives (such as stochastic thermal agitation or self-driving) or the slow drives, can lead to scale-free spatiotemporal fluctuations over a certain range of the control parameter, as experimentally and numerically demonstrated in many self-organized criticality (SOC)-type systems and interface fluctuations in passive biphase systems $[1-5,40]$. In the cell system, the selfdriven cell motions provide active drives to generate disorder. In our binary cell mixture, in addition to the aggregation of CCs due to the weakest CC-EC coupling, the self-driving of mobile $\mathrm{CCs}$ and weak $\mathrm{CC}-\mathrm{CC}$ coupling generate scale-free turbulent $\mathrm{CC}$ motions in $\mathrm{CC}$ clusters. They are the sources to drive the surrounding ECs with low motility and strong EC-EC coupling, and induce boundary fluctuations.

Namely, for the CC cluster boundary, the multiscale spatiotemporal fluctuations are determined by the interplay of CC turbulence due to high $\mathrm{CC}$ motility and low $\mathrm{CC}-\mathrm{CC}$ coupling, and surrounding ECs with low motility and strong EC-EC coupling, which suppress motion and structural rearrangement. Excited by the scale-free turbulentlike motion in the CC clusters, the CC cluster boundary structure affects the forces on the boundary, and in turn provides a feedback to determine the velocity and subsequent structure of the boundary. It is the key for self-consistently sustaining the correlated scale-free fluctuations of the structure and the velocity of the CC cluster boundary.

Through aggregation, the $\mathrm{CC}$ cluster with a larger size and more CCs facilitates longer-scale fluctuations. It causes the consistent increases of $D_{A}, p, \alpha$ (the scaling exponent of MSRV), and $\beta$ (the absolute value of the scaling exponent of CC velocity spectrum in Fig. S5) with increasing $A$. Their consistent faster decreases with increasing $t_{w}$ for $t_{w} \geqslant$ $30 \mathrm{~h}$ reflect the reduced complexities of CC turbulent motion, and boundary fluctuations both in structure and motion, by proliferation-induced crowding and dynamical slowing down. They also evidence the strong correlation of $\mathrm{CC}$ motion in the cluster, and the structure and motion of the fluctuating CC cluster boundary.

The above self-similar multiscale behaviors are akin to the scale-free SOC-type behaviors in certain ranges of control parameters of various nonequilibrium nonlinear extended systems under persistent or stochastic drives, such as avalanche cracking, seismic activities, and melting, wetting, and fire burning interfaces $[1-5,40]$. For binary cell mixtures, to our knowledge, previous works have not addressed the issues sim- 
ilar to ours about the multiscale cluster size distributions, the multiscale structural and dynamical behaviors of the cluster interfaces, and their correlations [10-17].

In conclusion, we have investigated the multiscale area, morphology, and dynamical behaviors of $\mathrm{CC}$ cluster aggregation, especially demonstrating the scale-free fluctuations in structure and motion of the $\mathrm{CC}$ cluster boundary, in the proliferating CC-EC binary mixture from the dilute to the confluent state. The higher $\mathrm{CC}$ motility than EC motility and the weaker $\mathrm{CC}$-EC coupling cause $\mathrm{CC}$ aggregation into clusters exhibiting turbulentlike motion with a finite correlation length. The gradual merging of single $\mathrm{CCs}$ and $\mathrm{CC}$ clusters with different sizes and motion coherence causes the transition from the stretched exponential to the scale-free power-law distributions of the cluster area. The interplay of the multiscale $\mathrm{CC}$ turbulent motion and the surrounding less mobile and more strongly coupled ECs through the fluctu- ating boundary, is the key to self-consistently sustain the correlated scale-free multiscale fluctuations of the structure and velocity of $\mathrm{CC}$ cluster boundaries. Increasing cluster area facilitates stronger fluctuations with increasing length scale and increases the scaling exponents of velocity as well as position fluctuations of $\mathrm{CC}$ cluster boundaries, which can be suppressed by proliferation-induced crowding and dynamical slowing down.

This study paves a way for investigating and understanding the generic dynamical behaviors of aggregation and multiscale interface fluctuations in various active binary systems, an important issue comparing with the widely studied interface fluctuations of various passive binary mixtures.

This work is supported by the Ministry of Science and Technology of Taiwan, under Contract No. MOST-109-2112M-008-007.
[1] A.-L. Barabási and H. E. Stanley, Fractal Concepts in Surface Growth (Cambridge University Press, New York, 1995); T. Bohr, M. H. Jensen, G. Paladin, and A. Vulpiani, Dynamical Systems Approach to Turbulence (Cambridge University Press, Cambridge, UK, 1998).

[2] M. Rubin-Zuzic, G. E. Morfill, A. V. Ivlev, R. Pompl, B. A. Klumov, W. Bunk, H. M. Thomas, H. Rothermel, O. Havnes, and A. Fouquét, Kinetic development of crystallization fronts in complex plasmas, Nat. Phys. 2, 181 (2006).

[3] W. Wang, H. W. Hu, and L. I, Surface-Induced Layering of Quenched 3D Dusty Plasma Liquids: Micromotion and Structural Rearrangement, Phys. Rev. Lett. 124, 165001 (2020).

[4] S. He, G. L. M. K. S. Kahanda, and P.-Z. Wong, Roughness of Wetting Fluid Invasion Fronts in Porous Media, Phys. Rev. Lett. 69, 3731 (1992).

[5] J. Zhang, Y.-C. Zhang, P. Alstrøm, and M. T. Levinsen, Modeling forest fire by a paper-burning experiment, a realization of the interface growth mechanism, Physica A 189, 383 (1992).

[6] T. A. Witten, Jr. and L. M. Sander, Diffusion-Limited Aggregation, a Kinetic Critical Phenomenon, Phys. Rev. Lett. 47, 1400 (1981).

[7] A. E. Patteson, A. Gopinath, and P. E. Arratia, The propagation of active-passive interfaces in bacterial swarms, Nat. Commun. 9, 5373 (2018).

[8] D. H. Rothman, Deformation, Growth, and Order in Sheared Spinodal Decomposition, Phys. Rev. Lett. 65, 3305 (1990).

[9] H. Tanaka, Double Phase Separation in a Confined, Symmetric Binary Mixture: Interface Quench Effect Unique to Bicontinuous Phase Separation, Phys. Rev. Lett. 72, 3690 (1994).

[10] D. A. Beysens, G. Forgacs, and J. A. Glazier, Cell sorting is analogous to phase ordering in fluids, Proc. Natl. Acad. Sci. USA 97, 9467 (2000).

[11] E. Méhes, E. Mones, V. Németh, and T. Vicsek, Collective motion of cells mediates segregation and pattern formation in co-cultures, PLoS One 7, e31711 (2012).

[12] C. Chatelain, T. Balois, P. Ciarletta, and M. Ben Amar, Emergence of microstructural patterns in skin cancer: A phase separation analysis in a binary mixture, New J. Phys. 13, 115013 (2011).
[13] E. Mones, A. Czirók, and T. Vicsek, Anomalous segregation dynamics of self-propelled particles, New J. Phys. 17, 063013 (2015).

[14] C. P. Beatrici, R. M. C. de Almeida, and L. G. Brunnet, Mean-cluster approach indicates cell sorting time scales are determined by collective dynamics, Phys. Rev. E 95, 032402 (2017).

[15] A. J. Kabla, Collective cell migration: leadership, invasion and segregation, J. R. Soc. Interface 9, 3268 (2012).

[16] J. M. Belmonte, G. L. Thomas, L. G. Brunnet, R. M. C. de Almeida, and H. Chaté, Self-Propelled Particle Model for CellSorting Phenomena, Phys. Rev. Lett. 100, 248702 (2008).

[17] C. Strandkvist, J. Juul, B. Baum, A. Kabla, and T. Duke, A kinetic mechanism for cell sorting based on local variations in cell motility, Interface Focus 4, 20140013 (2014).

[18] P. Friedl and D. Gilmour, Collective cell migration in morphogenesis, regeneration and cancer, Nat. Rev. Mol. Cell Biol. 10, 445 (2009).

[19] O. Ilina and P. Friedl, Mechanisms of collective cell migration at a glance, J. Cell Sci. 122, 3203 (2009).

[20] A. Labernadie et al., A mechanically active heterotypic E-cadherin/N-cadherin adhesion enables fibroblasts to drive cancer cell invasion, Nat. Cell Bio. 19, 224 (2017).

[21] D. T. Tambe et al., Collective cell guidance by cooperative intercellular forces, Nat. Mater. 10, 469 (2011).

[22] A. Haeger, M. Krause, K. Wolf, and P. Friedl, Cell jamming: Collective invasion of mesenchymal tumor cells imposed by tissue confinement, Biochim. Biophys. Acta 1840, 2386 (2014).

[23] H. Y. Chen, Y. T. Hsiao, S. C. Liu, T. Hsu, W. Y. Woon, and L. I, Enhancing Cancer Cell Collective Motion and Speeding up Confluent Endothelial Dynamics through Cancer Cell Invasion and Aggregation, Phys. Rev. Lett. 121, 018101 (2018).

[24] T. E. Angelini, E. Hannezo, X. Trepat, M. Marquez, J. J. Fredberg, and D. A. Weitz, Glass-like dynamics of collective cell migration, Proc. Natl. Acad. Sci. USA 108, 4714 (2011).

[25] D. Bi, J. H. Lopez, J. M. Schwarz, and M. L. Manning, A density-independent rigidity transition in biological tissues, Nat. Phys. 11, 1074 (2015). 
[26] J. A. Park et al., Unjamming and cell shape in the asthmatic airway epithelium, Nat. Mater. 14, 1040 (2015).

[27] S. Garcia, E. Hannezo, J. Elgeti, J. F. Joanny, P. Silberzan, and N. S. Gov, Physics of active jamming during collective cellular motion in a monolayer, Proc. Natl. Acad. Sci. USA 112, 15314 (2015).

[28] A. Hayer, L. Shao, M. Chung, L.-M. Joubert, H. W. Yang, F.-C. Tsai, A. Bisaria, E. Betzig, and T. Meyer, Engulfed cadherin fingers are polarized junctional structures between collectively migrating endothelial cells, Nat. Cell Biol. 18, 1311 (2016).

[29] B. Li and S. X. Sun, Coherent motions in confluent cell monolayer sheets, Biophys. J. 107, 1532 (2014).

[30] G. Duclos, C. Erlenkamper, J. F. Joanny, and P. Silberzan, Topological defects in confined populations of spindle-shaped cells, Nat. Phys. 13, 58 (2017).

[31] A. Puliafito, L. Hufnagel, P. Neveu, S. Streichan, A. Sigal, D. K. Fygenson, and B. I. Shraniman, Collective and single cell behavior in epithelial contact inhibition, Proc. Natl. Acad. Sci. USA 109, 739 (2012).

[32] A. Szabó, R. Ünnep, E. Méhes, W. O. Twal, W. S. Argraves, Y. Cao, and A. Czirók, Collective cell motion in endothelial monolayers, Phys. Biol. 7, 046007 (2010).

[33] S. E. Leggett, Z. J. Neronha, D. Bhaskar, J. Y. Sim, T. M. Perdikari, and I. Y. Wong, Motility-limited aggregation of mammary epithelial cells into fractal-like clusters, Proc. Natl. Acad. Sci. USA 116, 17298 (2019).
[34] W. K. Chang, C. Carmona-Fontaine, and J. B. Xavier, Tumour stromal interactions generate emergent persistence in collective cancer cell migration, Interface Focus 3, 20130017 (2013).

[35] J. W. Baish and R. K. Jain, Fractals and cancer, Persp. Cancer Res. 60, 3683 (2000).

[36] F. E. Lennon, G. C. Cianci, N. A. Cipriani, T. A. Hensing, H. J. Zhang, C.-T. Chen, S. D. Murgu, E. E. Vokes, M. W. Vannier, and R. Salgia, Lung cancer-a fractal viewpoint, Nat. Rev. Clin. Oncol. 12, 664 (2015).

[37] A. Chan and J. A. Tuszynski., Automatic prediction of tumour malignancy in breast cancer with fractal dimension, R. Soc. Open Sci. 3, 160558 (2016).

[38] See Supplemental Material at http://link.aps.org/supplemental/ 10.1103/PhysRevResearch.3.L032050 for more details about (a) experimental methods including cell incubation, PIV, CC cluster boundary identification and Lagrangian trajectory tracking, (b) turbulentlike motion in CC clusters, (c) significance of the fractal structure of $\mathrm{CC}$ cluster boundaries and scale-free motions of CCs and CC cluster boundaries, and (d) videos of phase-contrast images and Lagrangian trajectories of binary CC-EC mixture, and videos of the phase-contrast images of monotype CCs and ECs.

[39] W. Thielicke and E. J. Stamhuis, http://PIVlab.blogspot.com.

[40] P. Bak, How Nature Works-The Science of Self-Organized Criticality (Oxford University Press, Oxford, UK, 1977); H. J. Jensen, Self-Organized Criticality (Cambridge University Press, New York, 1998). 